Plant development

\section{Leaves by number}

Fibonacci numbers are notorious for appearing in the most unlikely places, including the architecture of plants. Elsewhere in this issue, Didier Reinhardt and colleagues describe the role of the plant hormone auxin in phyllotaxis, the positioning of leaves around a stem (Nature 426, 255-260; 2003). In so doing, they reveal a mechanism by which Fibonacci numbers can emerge.

Fibonacci was the nickname of the Italian mathematician Leonardo Pisano (1170-1250), who introduced the Arabic number system into Europe. In 1202, he also posed a seemingly inconsequential problem concerning the breeding of rabbits. Its solution required the use of a series of numbers whose members are the sum of the two preceding entries $(1,1,2,3,5,8$, $13,21,34,55, \ldots)$. This series now bears his name.

The Fibonacci series occurs in the arrangement of many plant organs. The seeds of sunflowers, Helianthus annuus, and the leaves of cacti and succulents (such as Mammillaria myrtax, upper image here, and Sempervivum hybrida, lower image) are arranged in both left- and right-handed spirals. The numbers of leaves, or seeds, in these spirals are consecutive Fibonacci numbers.

Leaves are also spirally distributed around the stems of less exotic plants. Here they tend to be separated by an angle of $137.5^{\circ}$. This is the radial equivalent of the golden ratio, $\approx 1.618$, the ultimate proportional increase between successive Fibonacci numbers. Many hypotheses, ranging from the prosaic to the mystical, have been proposed to explain why leaves should stick out at this angle. Some invoke physical properties of the stem, whereas others propose that growing leaves emit an inhibitory field to prevent new leaves from arising in their vicinity, but none has had direct supporting evidence.

Leaves originate at the tips of growing shoots in a self-renewing region known as the shoot apical meristem. Using the thale cress, Arabidopsis thaliana, Reinhardt and colleagues saw that new leaves formed where the concentration of auxin was highest. Indeed, artificially adding auxin to specific points on the surface of the meristem caused leaves to be produced at those points.

Auxin is a growth stimulator that is propelled through plant tissues by specific influx and efflux transporters. Reinhardt et al. looked at the distribution of one of the most important of these, the efflux protein PIN1, as well as mutants in which it was missing, and deduced that auxin is moved towards the shoot tip

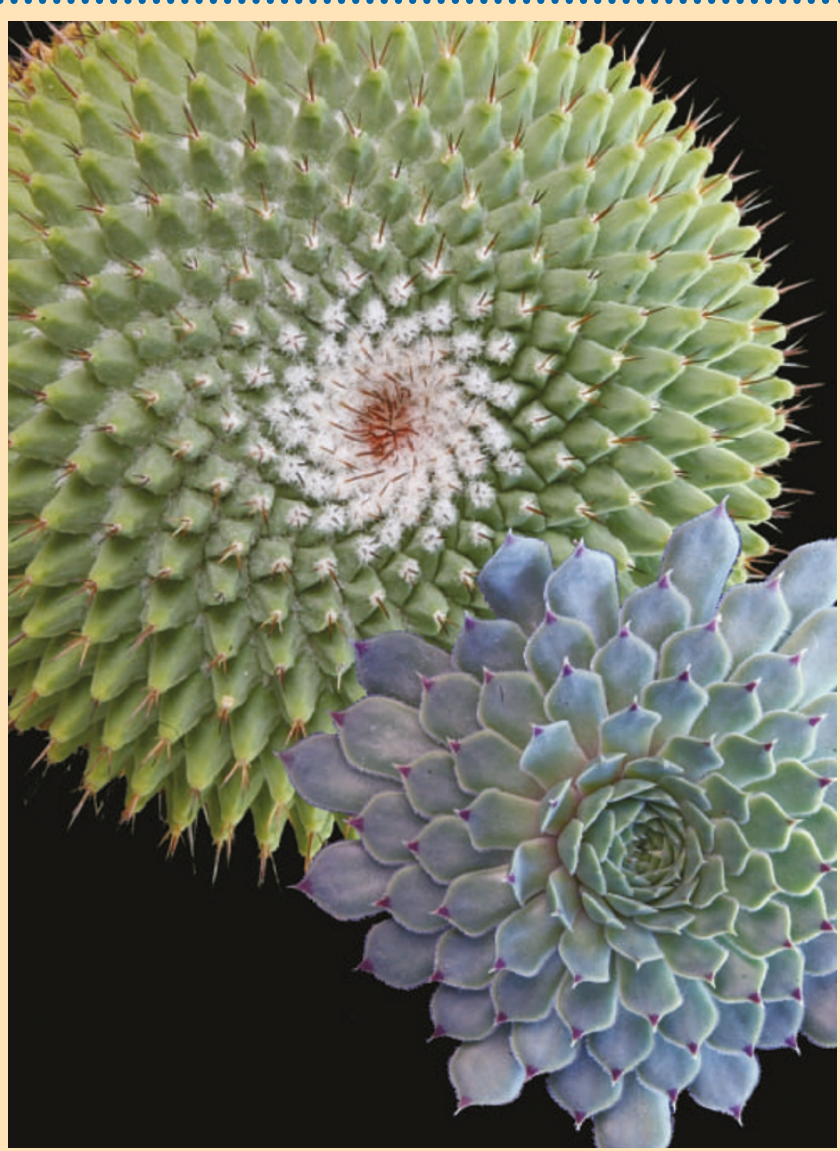

through the outer layers of the shoot apical meristem. Existing leaf buds act as sinks, preventing auxin from continuing its progress directly above them. The maximum auxin concentration, and so the site of new leaf formation, is thus as far away as possible from already-formed leaves.

Reinhardt et al. have therefore uncovered a mechanism in which neither by a physical property of the stem, nor by an inhibitory field produced by growing leaves. Instead, the gaps between leaves, by allowing the free flow of auxin, mark out the position of each new leaf.

Christopher Surridge the position of leaves is determined itself or by a stellar companion. Increasingly, observations and calculations are pointing to stellar companions as the source of such jets.

Many so-called symbiotic binary systems, which contain a red-giant star and a smaller stellar companion, have bipolar nebulae around them that are remarkably similar to some planetary nebulae. This suggests that binary companions could be at the root of the shaping process in both symbiotic nebulae and planetary nebulae ${ }^{4}$. Some symbiotic systems are known to have jets ${ }^{5,6}$, and the newly found jets near red-giant stars $^{1,2}$ strongly support jet shaping of some planetary nebulae as well. Sahai and colleagues' discovery ${ }^{1}$ is particularly suggestive on this point: the wind speed of the jet from around V Hydrae is much higher than the value expected for material ejected from giant stars; so it seems more likely that the jet is emanating from a much smaller stellar companion as it accretes matter from the dying star. Furthermore, V Hydrae is known to rotate more rapidly than expected, suggesting that its rate of spin is being affected by a companion star.

The existence of jets in planetary nebulae may also help to explain the similarity between bipolar structures in some planetary nebulae and those in some clusters of galaxies - even though the latter are a million times larger. Both can appear to have oppositely balanced pairs of bubbles in their structure (Fig. 1). In galaxy clusters, these are known to be formed by two oppositely ejected jets. The new finding ${ }^{1}$, then, brings us closer to describing a unified formation mechanism for pairs of bubbles in astrophysical systems, over many orders of magnitude in system size.

Stimulating and significant as this jet discovery is, some links are still missing. The collimated flow detected by Sahai et al. ${ }^{1}$ is less than three years old - is there also a long-lived jet in that direction, or does this indicate that mass loss is sporadic rather than continuous? A second jet to balance this one would also be expected. As Sahai et al. state, the counter-jet may be obscured by dense gas, but it or its signature should now be sought. And, of course, efforts must be made to find the stellar companion, the suspected source of the jets that will shape the planetary nebula of V Hydrae.

Noam Soker is in the Department of Physics,

Technion - Israel Institute of Technology,

Haifa 32000, Israel.

e-mail:soker@physics.technion.ac.il

1. Sahai, R., Morris, M., Knapp, G. R., Young, K. \& Barnbaum, C. Nature 426, 261-264 (2003).

2. Imai, H., Obara, K., Diamond, P. J., Omodaka, T. \& Sasao, T. Nature 417, 829-831 (2002).

3. Claussen, M. Nature 417, 798-799 (2002).

4. Corradi, R. L. M. \& Schwarz, H. E. Astron. Astrophys. 293, 871-888 (1995).

5. Kellogg, E., Pedelty, J. A. \& Lyon, R. G. Astrophys. J. 563, L151-L155 (2001).

6. Brocksopp, C. et al. Mon. Not. R. Astron. Soc. (in the press). 